\title{
Acute Lepromatous Ulceration of the Skin
}

\author{
A. B. A. KARAT, B.Sc., M.B.B.S., M.R.C.P. (LOND.), M.R.C.P. (EDIN.) \\ C. K. JOB, B.SC., M.D., M.C. PATH. \\ Mrs. S. KARAT, M.B.B.S., F.R.C.S. (EDIN.) \\ Schieffelin Leprosy Research Sanatorium, Karigiri, via Katpadi, N.A. Dist., S. India
}

A great deal has been written regarding the exacerbated phases in the various types of leprosy. Recently Tolentino (1965) presented an excellent summary of the clinical features of acute manifestations of leprosy at the research conference in Washington. A comprehensive discussion on exacerbated phases in leprosy can also be found in the report of the panel on reaction at the VIII International Congress of Leprology (Rio, 1963).

It is generally agreed that there are three fairly well defined groups of acute manifestations in lepromatous leprosy (Cochrane, 1964; Tolentino, 1965; Tijiri, 1955), namely:-

(1) Acute Lepromatous Infiltration or acute exacerbation of lepromatous leprosy during which the disease progresses rapidly. At this stage there may be striking increase in generalised infiltration of the skin with thickening of the skin and erythema; in some others, infiltrated plaque-like lesions with sloping edges and/or nodules may appear, especially over the face and ears.

(2) Erythema Nodosum Leprosum which is characterised by the appearance of painful, erythematous nodular eruption usually associated with systemic symptoms such as fever, anorexia, arthralgia and swelling of the feet; and

(3) The Lucio Phenomenon which is a special manifestation reported almost exclusively from Latin American countries. 'It is described ..s a necrotising vasculitis beginning as purpuric lesions that become necrotic in the centre and break down into ulcers. It is observed chiefly in advanced cases with diffuse lepromatous infiltration.' (Tolentino, 1965).

We are here describing another acute manifestation in lepromatous leprosy hitherto in- adequately documented. This is characterised by an abrupt spread of lepromatous infiltrated lesions which quickly ulcerate in the centre of the lepromatous plaque or nodule and the patient presents with a large number of skin ulcers with very little systemic disturbance.

Patient No. 1-R.R.: S.I.R.S. 7861 . Male aged 45 years; was first seen at S.L.R.S. on 2.11.65 with a 7-year history of lepromatous leprosy. One month prior to arrival at S.L.R.S., there was a sudden flare up of the disease with appearance of new raised lesions over the trunk and limbs, some of which ulcerated. There was no other systemic symptom.

On Examination: The patient was found to have advanced lepromatous leprosy with loss of eyebrows, enlargement of ears, early depression of nose, gross infiltration of the skin from head to foot, and bilateral ulnar paralysis. There were a large number of infiltrated, raised lesions with elevated centres and sloping edges, of variegated size and shape (Figs. 1 and 2). A number of these lesions had ulcerated in the centre, discharging a sero-sanguinous material which, on acid fast staining, showed sheets of uniformly staining acid fast rods and globi filled with acid fast bacilli.

Patient was treated with intramuscular injections of Streptomycin $1.0 \mathrm{~g}$. daily and INAH $300 \mathrm{mg}$. once a day. The skin lesions healed within a week (Figs. 3 and 4). There has been a rapid improvement in the clinical condition of the patient and simultaneously the bacterial index has come down very dramatically.

Skin Smears:

\begin{tabular}{rcc}
\hline \multicolumn{1}{c}{ Date } & B. Index & \% Rods \\
\hline 2.11 .65 & 5.0 & 75 \\
17.12 .65 & 3.5 & 10 \\
8.2 .66 & 2.0 & \\
25.4 .66 & 1.0 & \\
20.6 .66 & 0.87 & 2 \\
\hline
\end{tabular}

Bone Marrow: Aspirate showed groups of rod shaped acid fast bacilli on 4.11.65. On 20.6.66 no bacilli could be found in the bone marrow aspirate. 
Skin Biopsy: Showed the epidermis atrophic with flattening of the rete pegs. There was a clear area beneath the epidermis separating a band of inflammatory cells consisting of mostly foamy macrophages. There were also scattered collections of lymphocytes. An occasional plasma cell was present.

Acid fast stain showed numerous bacilli inside macrophages.

This picture was that of a patient with advanced lepromatous leprosy.

Patient No. 2-S.N.P.: S.L.R.S. 8053. Male aged 48 years; was admitted here on 9.3 .66 with a 10 -year history of leprosy which began with a patch on the thigh. He had taken treatment irregularly. About 2 months prior to arrival here there was a sudden flare up of the disease with the appearance of raised lesions all over the body which quickly went on to ulcerate in the centre.

On Examination there was gross lepromatous infiltration of the skin from head to foot; partial loss of eyebrows and gross oedema of the legs and feet. There were innumerable skin ulcers in various stages of evolution. Majority of these ulcers were in the middle of lepromatous, infiltrated, raised lesions (Figs. 5 and 6 ). Both the eyes were congested, there was corneal haziness, circumcorneal injection and yellowish-white nodules on the conjunctiva near the limbus on both sides. There was marked bilateral gynaecomastia, and both testes were atrophic.

Patient was treated with 1.0 g. of Streptomycin daily, and $300 \mathrm{mg}$. INAH once a day orally. In 14 days all skin ulcers healed, leaving papery thin scars (Figs. 7 and 8). Along with the striking clinical improvement, the skin bacillary index also came down rapidly.

Skin Smears:

\begin{tabular}{rcc}
\hline Date & B. Index & \% Rods \\
\hline 10.3 .66 & 4.87 & 60 \\
31.3 .66 & 3.62 & 25 \\
19.4 .66 & 3.37 & 5 \\
10.6 .66 & 3.25 & \\
8.8 .66 & 2.62 & 5 \\
\hline
\end{tabular}

Bone Marrow: Aspirate on admission to S.L.R.S., had acid fast bacilli in rod form.

Skin Biopsy: Epidermis showed some proliferation of the prickle cells. There was marked parakeratosis and some hyperkeratosis. Beneath the epithelium was seen a dense inflammatory infiltrate composed almost entirely of foamy macrophages. There were also scattered lymphocyctes and plasma cells. In one area there was an ulcer, the superficial part of which was formed by granulation tissue consisting of capillaries, polymorphonuclear leucocytes, lymphocytes and plasma cells. In the deeper area there were large collections of foamy macrophages.
On acid fast stain numerous bacilli inside macrophages were seen.

This picture was quite consistent with a lepromatous ulcer.

Patient No. 3-Y:S.L.R.S. 7917. Femaleaged 25 years; was first seen here on 6.12 .65 with a 10 -year history of leprosy which began with a hypopigmented patch over left arm. About 3 months ago, there was an abrupt onset of extensive skin ulceration without any systemic disturbance.

On Examination there was gross infiltration of the skin from head to foot. Thrre was enlargement of ears; early depression of the nose; and partial loss of eyebrows. There were extensive areas of skin ulceration over the trunk, limbs and face (Figs. 9 and 10) discharging sero-sanguinous fluid which on acid fast staining showed numerous rods and globi. Treatment was begun with daily injections of $1.0 \mathrm{~g}$. Streptomycin and oral INAH $300 \mathrm{mg}$. once a day and in 3 weeks time all the skin ulcers healed leaving papery thin scars (Figs. 11 and 12). Along with the healing of ulcers and rapid resolution of the gross skin infiltration, the skin bacillary index came tumbling down.

Skin Smears:

\begin{tabular}{rcc}
\hline \multicolumn{1}{c}{ Date } & B. Index & $\%$ Rods \\
\hline 6.12 .65 & 4.62 & 95 \\
4.1 .66 & 3.87 & 60 \\
18.1 .66 & 3.87 & 55 \\
9.3 .66 & 3.75 & \\
23.5 .66 & 3.00 & 3 \\
26.7 .66 & 2.05 & \\
\hline
\end{tabular}

Bone Marrow: Acid fast bacilli in rod form seen in aspirate.

Skin Biopsy: The histological picture of the skin in this case was very similar to what was described under Patient No. 2.

The Liver Biopsy section showed small focal collections of foamy macrophages containing acid fast bacilli.

Patient No. 4-D: S.L.R.S. 7918 (son of Y. 7917), aged 10 years; seen at S.L.R.S. on 6.12 .65 for the first time with a 4-year history of leprosy. During the last 1 year the disease spread rapidly and enlargement of the ears was noticed. About 3 months ago a number of the raised skin lesions ulcerated and would not heal.

On Examination there was gross, coarse infiltration of the skin from head to foot; enlargement of ears with nodules along the pinna; a number of raised, infiltrated lesions with sloping edges and raised centre of varying sizes and shapes all over the body, face and limbs; partial loss of eyebrows (Fig. 13). Many of the infiltrated lesions had ulcerated discharging sero-sanguinous fluid which on acid fast staining was full of rods and globi. Patient was started on treatment with daily injections of $0.5 \mathrm{~g}$. of Streptomycin and $150 \mathrm{mg}$. of INAH orally. The skin ulcers healed in 2 weeks (Fig. 14). 


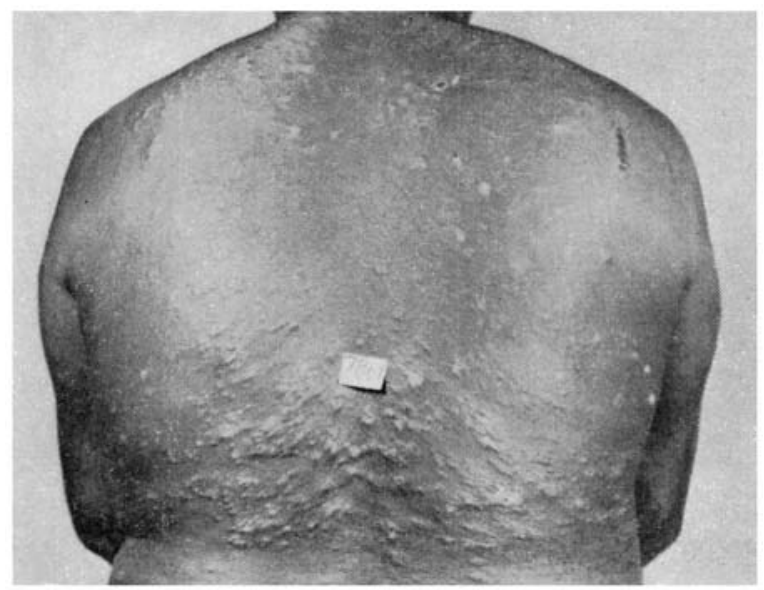

FIG. 1

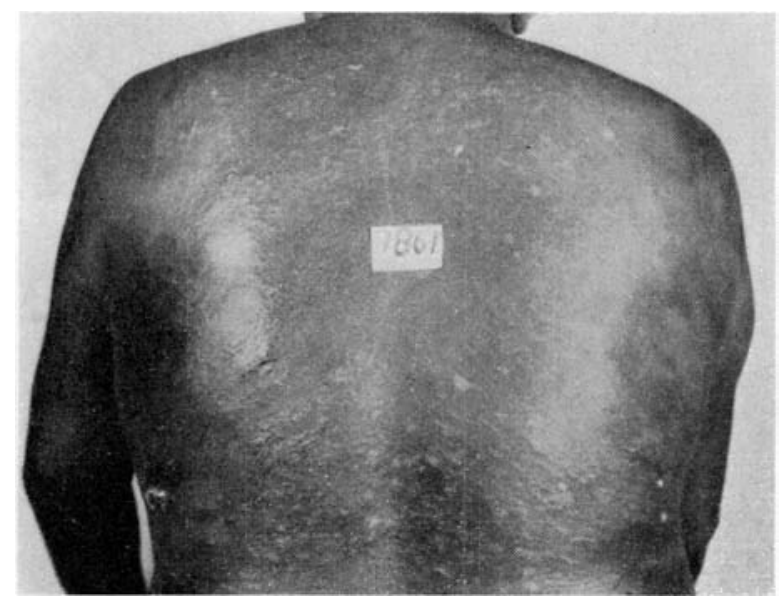

Fig. 3

FIG. 1. Shows the back on admission. Notice the infiltrated lesions on the skin, some of which have ulcerated.

FIG. 2. To show oedema of the ankles and superficial skin ulceration.

FIcs. 3 and 4. Same patient as in 1 and 2-after treatment. The skin ulcers have healed and the raised, infiltrated lesions have flattened out.

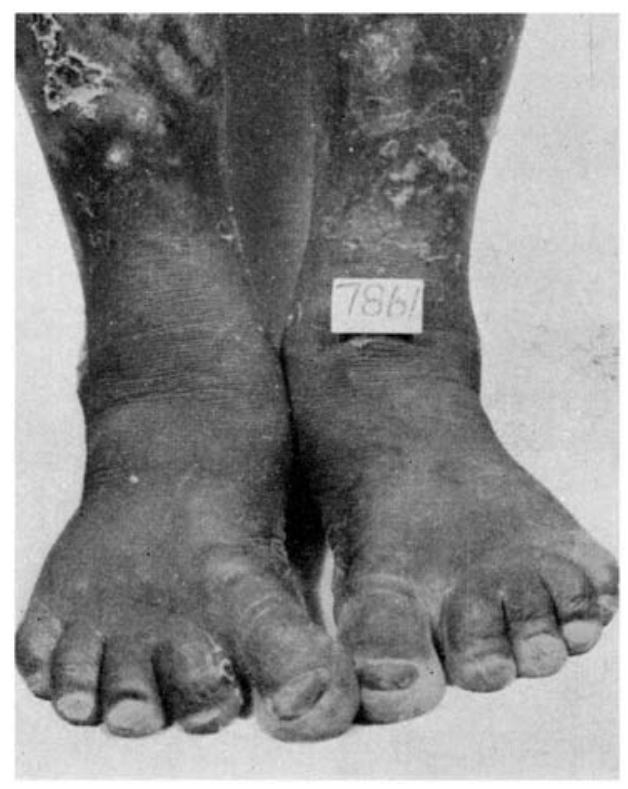

FIG. 2

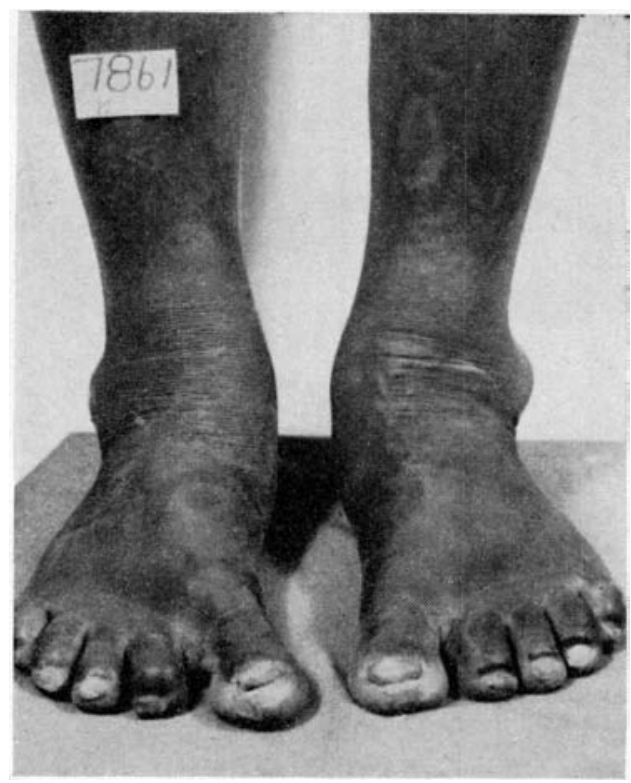

Fig. 4 


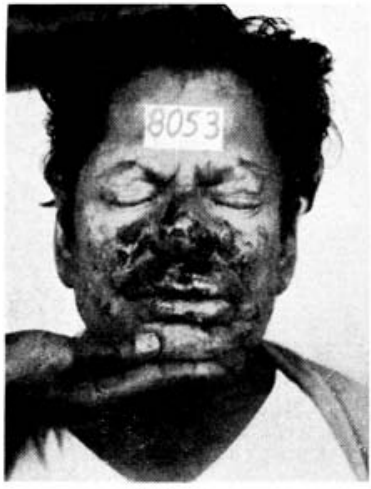

FiG. 5

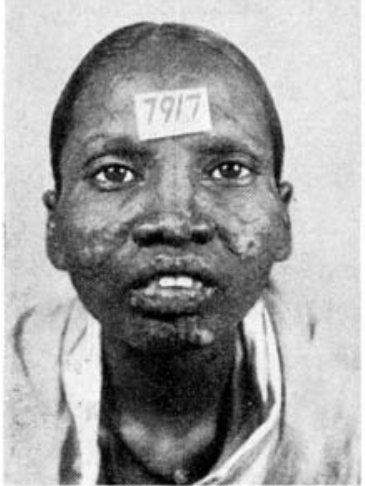

FIG. 9

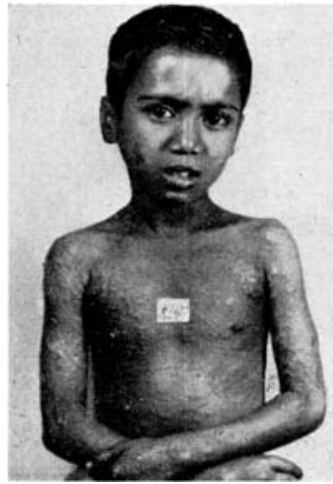

FIG. 13

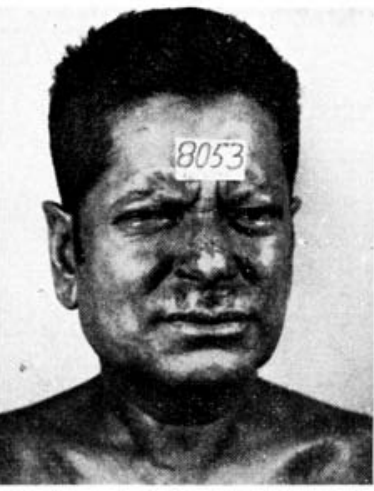

FIG. 6

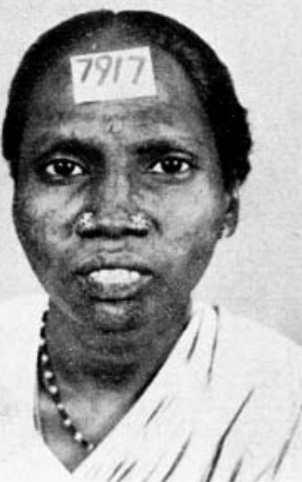

FIG. 10

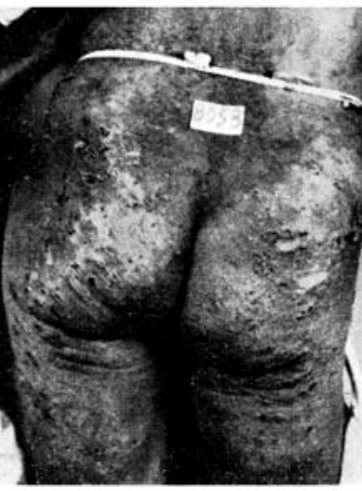

FIG. 7

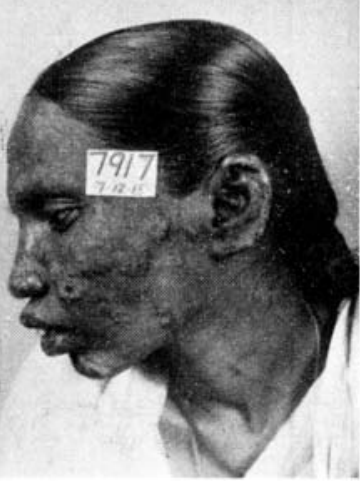

FIG. 11

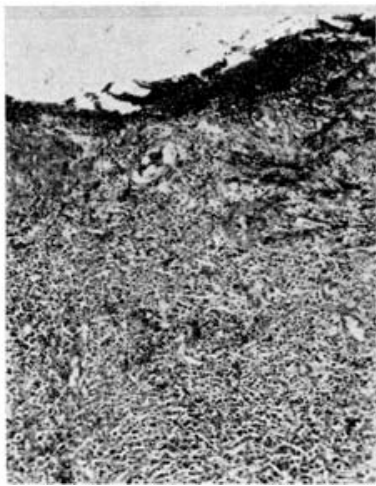

FIG. 15

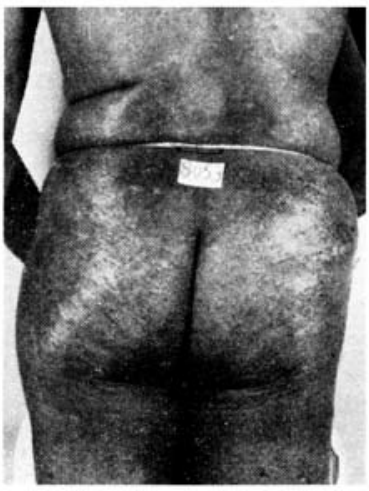

FIG. 8

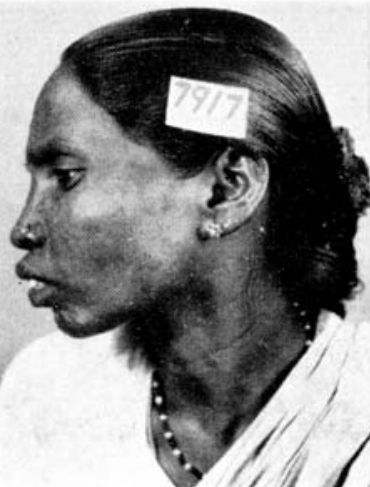

FIG. 12

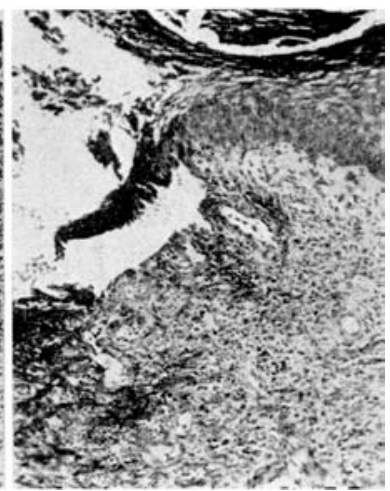

FIG. 16

FIg. 13. Before treatment-gross lepromatous infiltration and areas of ulceration are seen.

FIG. 14. After treatment-the clinical improvement is easily appreciated.

FIG. 15. Photomicrograph to show the edge of the lepromatous ulcer.

FIG. 16. The bed of the ulcer consists of a superficial layer of necrosis, a layer of inflammatory cells consisting of lymphocytes, macrophages and a few polymorphs and then a sheet of foamymacrophagescontaining acid fast bacilli. 
S'kin Smears:

\begin{tabular}{rcc}
\hline \multicolumn{1}{c}{ Date } & B. Index & \% Rods \\
\hline 6.12 .65 & 4.62 & 90 \\
4.1 .66 & 3.62 & 58 \\
18.1 .66 & 3.62 & 48 \\
21.5 .66 & 2.75 & 2 \\
\hline
\end{tabular}

Bone Marrow: Acid fast bacilli in rod form seen in aspirate.

Skin Biopsy: Section shows a lepromatous granuloma of the skin with sheets of foamy macrophages underneath the epidermis. The inflammatory cell collections extend right up to the epidermis from which it is separated only by the basement membrane. There was no clear area between the epidermis and the granuloma.

Acid fast stain shows numerous bacilli inside macrophages.

\section{COMMENTS}

Practically everyone working in the field of leprosy would have seen patients with lepromatous leprosy in whom the disease for some reason or other suddenly begins to spread, and sometimes these lepromatous lesions ulcerate. Though one is familiar with this kind of clinical picture, we are unable to find a good description of this condition, nor any guide lines regarding management.

That these ulcers are directly a consequence of the proliferation of Mycolacterium leprae there is little doubt (Figs. 15 and 16). What was surprising was the rapidity of resolution of these lesions under treatment with Streptomycin and INAH, pari passu with corresponding drop in bacterial index.

In the patients here described, skin biopsies confirmed the presence of lepromatous granuloma in the affected area of skin. In those in whom liver biopsy had been done, there was the typical lepromatous granulomata and acid fast bacilli in the parenchymal tissue. Bone marrow aspirate also had typical acid fast bacilli one associates with lepromatous leprosy.

We would suggest that this particular behaviour pattern of the bacillus $M$. leprae may be due to 2 factors: (a) alteration in host immunity or $(b)$ mutation of the $M$. leprae. We are inclined to support the latter suggestion since the infection responds so dramatically to Streptomycin and INAH and the bacterial clearance apparently occurs concurrently with the clinical improvement in the physical condition of the patient, unlike the usual infection with $M$. leprae.

We would strongly recommend that this kind of patient with lepromatous skin ulceration should be treated with 1 g. of Streptomycin intramuscularly daily and $300 \mathrm{mg}$. of INAH orally once a day since in our limited experience this treatment brings about rapid healing of the ulcerated skin lesions concurrently with accelerated clearance of the dead bacilli. This response may in part be due to the bacteriocidal action of Streptomycin and increased susceptibility of this strain of $M$. leprae to Streptomycin.

SUMMARY

1. Four patients with acute lepromatous skin ulceration are described, with clinical and histopathological data.

2. Acute lepromatous skin ulceration presents with a history of abrupt spread of lepromatous leprosy lesions and infiltrations all over the body which rapidly progresses to skin ulceration, without any other systemic symptoms.

3. We recommend that patients with acute lepromatous skin ulceration be treated with Streptomycin 1 g. intramuscularly daily and INAH (isonicotinic acid hydrazide) $300 \mathrm{mg}$. once a day orally. These patients respond to this regime dramatically both as far as skin ulceration is concerned, as well as their bacteriological index on skin smear.

4. The possibility of this complication being due to a mutant strain of $M$. leprae is discussed. ACKNOWLEDGEMENTS

We are grateful to Mr. S. Jesudass for technical assistance with tissue sections; Mr. C. Dorairaj for photographs; and Mrs. L. Furness for secretarial help.

BIBLIOGRAPHY

1. cochrane, R. G. (1964). Leprosy in Theory and Practice. John Wright of Bristol. P. 336.

2. Report of the VIII International Congress of Leprology (Rio, 1963).

3. TAJIRI, I. (1955). The Acute Infiltration Reaction of Lepromatous Leprosy. Internat. J. Lep., 23, 371, 1955.

4. tolentino, J. G. (1965). Acute Manifestations of Leprosy. Internat. J. Lep., 33, 570, 1965.

Lepromatous Ulceration of the Skin 